\title{
Identifying Ancestral Origin using a Novel Panel of Single Nucleotide Polymorphism Locations
}

\author{
Zachary D. Williams \\ West Virginia University
}

Follow this and additional works at: https://researchrepository.wvu.edu/etd

\section{Recommended Citation}

Williams, Zachary D., "Identifying Ancestral Origin using a Novel Panel of Single Nucleotide Polymorphism Locations" (2012). Graduate Theses, Dissertations, and Problem Reports. 4937.

https://researchrepository.wvu.edu/etd/4937

This Thesis is protected by copyright and/or related rights. It has been brought to you by the The Research Repository @ WVU with permission from the rights-holder(s). You are free to use this Thesis in any way that is permitted by the copyright and related rights legislation that applies to your use. For other uses you must obtain permission from the rights-holder(s) directly, unless additional rights are indicated by a Creative Commons license in the record and/ or on the work itself. This Thesis has been accepted for inclusion in WVU Graduate Theses, Dissertations, and Problem Reports collection by an authorized administrator of The Research Repository @ WVU. For more information, please contact researchrepository@mail.wvu.edu. 


\title{
Identifying Ancestral Origin using a Novel Panel of Single Nucleotide Polymorphism Locations
}

\author{
Zachary D. Williams \\ Thesis submitted to the \\ Statler College of Engineering and Mineral Resources \\ at West Virginia University \\ in partial fulfillment of the requirements \\ for the degree of \\ Master of Science \\ in \\ Electrical Engineering
}

Donald Adjeroh, Ph.D., Chair

Arun Ross, Ph.D.

Xin Li, Ph.D.

The Statler College of Engineering and Mineral Resources

Morgantown, West Virginia

2012

Keywords: Ancestral Origin, Ear Size, SNPs

Copyright 2012 Zachary D. Williams 


\title{
Abstract \\ Identifying Ancestral Origin using a Novel Panel of Single Nucleotide Polymorphism Locations
}

\author{
Zachary D. Williams
}

A novel panel of single nucleotide polymorphisms (SNPs) used in the classification of ancestral origin is proposed. The panel is motivated by recent results in human identification and categorization using soft biometrics. The panel makes use of SNPs related to the height and ear size of an individual. The classifier proposed in this thesis is a standard maximum-likelihood classifier that matches an individual to the ancestral profile closest to the individual. This method produces results with lower error rates than other proposed methods.

This thesis proposes a new distinct panel of 55 single nucleotide polymorphisms for determining ancestral origin. This panel was later reduced to two panels of 18SNPs and 22SNPs respectively. This new approach is producing a high success rate, and has great promise for future progress. This panel is unique in that all prior research has made use of SNPs related to skin pigmentation since this is considered the most readily identifiable difference between different ancestral groups. We chose to use the four common ancestry groups of African American, Asian, European, and Sub-Saharan African.

The proposed panel of 55 SNPs had a successful classification rate of $98 \%$ when tested on simulated data, while the 22 SNP panel when tested on real data had a successful classification rate of $86 \%$ due to SNP availability issues in the real data. Using a knockout procedure, the 55 SNP panel was reduced to an 18 SNP panel that had a successful classification rate of $99.8 \%$ on simulated data. 


\section{Acknowledgement}

I would like to thank everyone who has contributed to my life over the last two years.

To my parents, thank you for helping me along the way and being a constant source of support and inspiration.

To Dr. Adjeroh, thank you for all of the assistance and inspiration while working on this project. You have pushed me to greater levels than I ever thought possible.

To my Committee Members: Dr. Ross and Dr. Li.

Thank you for all that you have shown me, leading to newer and better methods in furthering my own research.

This work was supported by a grant for CITeR-Codesign Initiative from the National Institute of Justice. 


\section{Table of Contents}

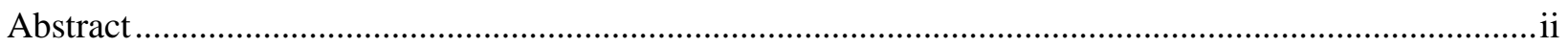



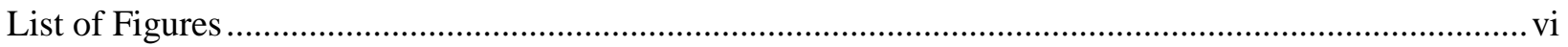

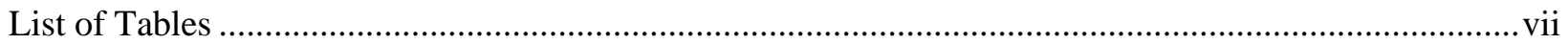

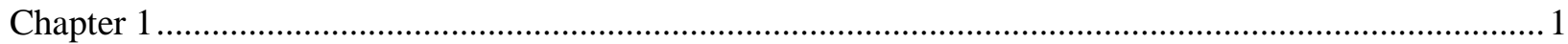



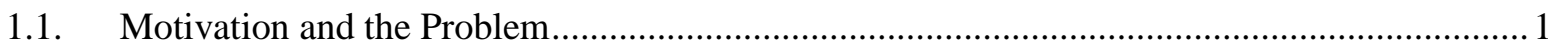

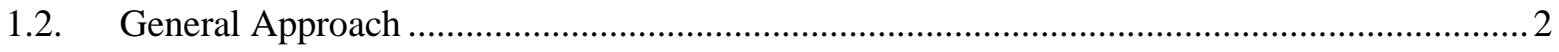

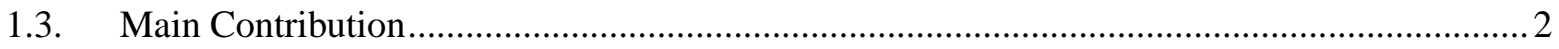

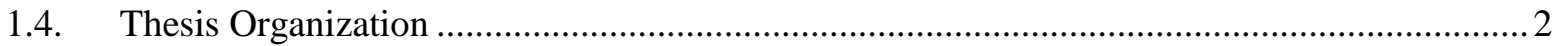

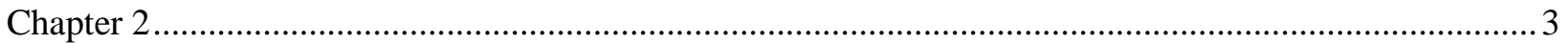

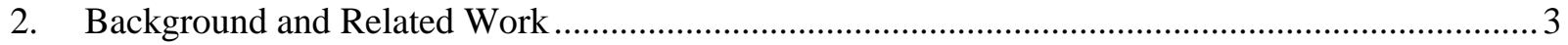

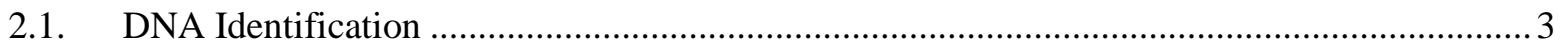

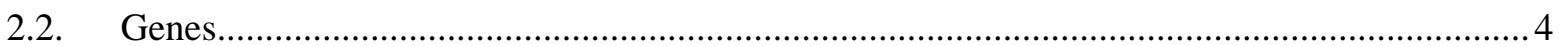



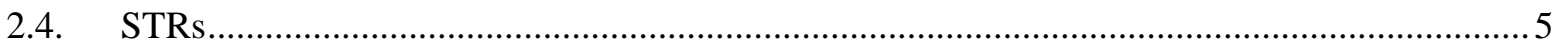

2.5. mtDNA

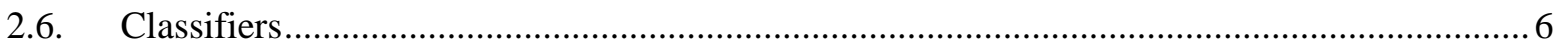

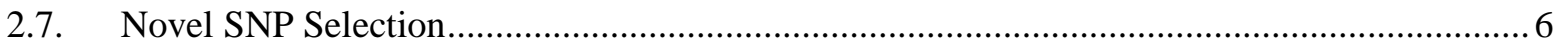

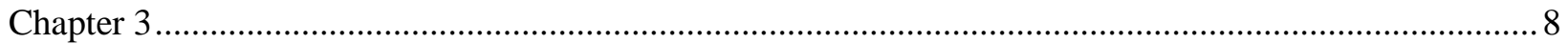

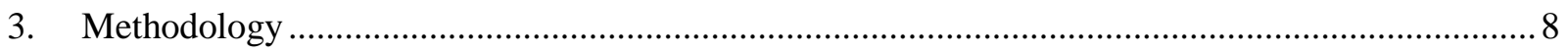

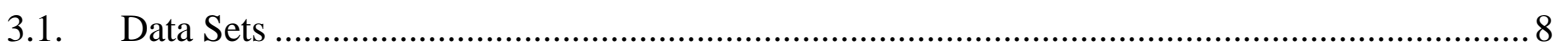

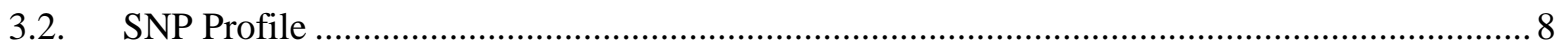

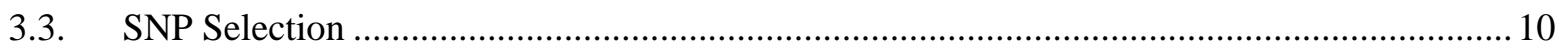

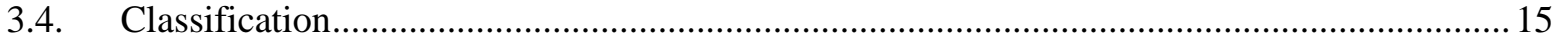

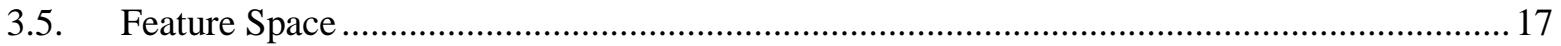

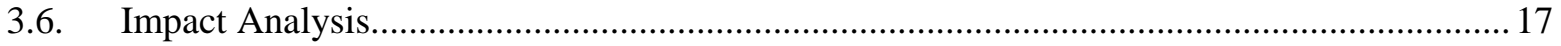



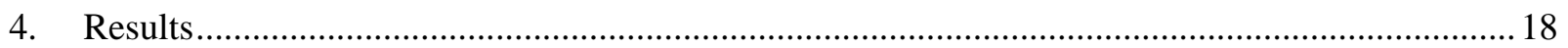

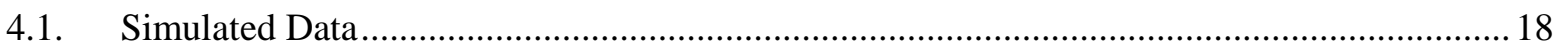

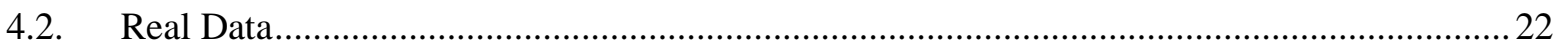








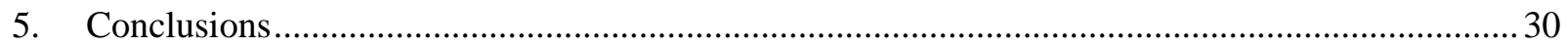

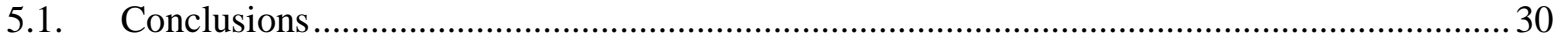



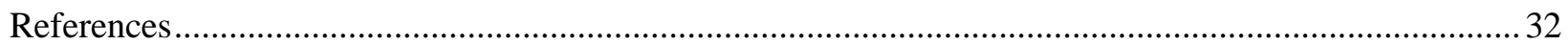




\section{List of Figures}

Figure 1: Frequency profile for the four ancestry groups for 3 SNPs. (a) rs2518881 (ear size) (b) rs29941 (weight) (c) rs849141 (height). The following notations are used: AA-African American; AAsian; E-European; SSA-Sub-Saharan African

Figure 2: Distance versus correlation plot used for possible SNP panel selection. ................................... 11



Figure 4: Error Curve used to find the SNP Panel with the lowest error............................................... 14

Figure 5: Using the SVM decision tree for multi-group classification. .................................................. 17

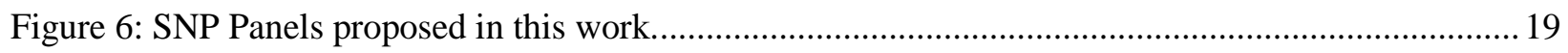

Figure 7: The amount of error for each ancestral group with different perturbation rates.......................20

Figure 8: Results of pair-wise comparisons between different ancestry groups using the proposed panel of



Figure 9: Results of pair-wise comparisons between different ancestry groups using the proposed panel of

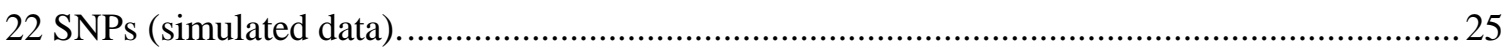

Figure 10: Results of pair-wise comparisons between different ancestry groups using real data, based on






\section{List of Tables}

Table 1: Dataset Summary of the Entrez Dataset. ................................................................................... 8

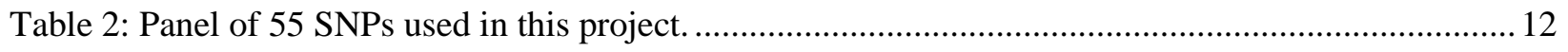

Table 3: Panel of 18 SNPs found after the impact analysis................................................................. 14

Table 4: Single SNP knockout results, 55 SNPs. (Top 20 SNPs with most impact) ...............................21

Table 5: Double SNP knockout results, 55 SNPs. (Top 20 SNP pairs with most impact) ........................22



Table 7: Single SNP knockout results, 22 SNPs (Top 20 SNPs with most impact)...............................26

Table 8: Double SNP knockout results, 22 SNPs (Top 20 SNP pairs with most impact). .......................27

Table 9: Triple SNP knockout results, 22 SNPs (Top 20 SNP triplets with most impact)......................28

Table 10: Comparative results of the different methods described in this project..................................29 


\section{Chapter 1}

\section{Introduction}

The practice of identifying an individual from their DNA was used in the early 80's and has been in use ever since. Ancestral identification did not gain wide acceptance until the new millennium when different groups of researchers came to the conclusion, see for example, $[1][2][3]$ that ancestral origin could be determined from DNA. Ancestral identification refers to determining where, geographically, an individual's ancestors come from.

\subsection{Motivation and the Problem}

The field of biometrics deals with the use of human physiological traits to recognize or identify individuals. Traditional hard biometric attributes such as fingerprints, face, iris, retinal scans, and vein patterns are well studied and have been quite successful in human recognition and identification [4].

More recently, it has been observed that other human attributes such as hair color, age, height, skin color, weight, etc also provide soft information which can be helpful in human recognition and categorization. Individually, each may not be enough to clearly discriminate between people. However, when combined, they could provide a powerful feature space for effective identification. These so-called soft biometrics [5] have become an area of intensive study in biometrics. In recent work, it was shown that soft biometric features such as wholebody human metrology [6] could be used to predict human attributes such as gender. Soft biometrics have been used to predict various human attributes, such as age [7], body weight [6],

and gender [8]. In another work, it has been shown that ear characteristics such as ear shape and ear size could provide important soft-biometric information that can aid human categorization or identification [9]. See also [10].

We were thus motivated to make soft-biometric attributes the focal point of our search for new Single Nucleotide Polymorphisms (SNPs) suitable for grouping people into their ancestral 
origins. That is, rather than following the traditional approach of using SNPs or Short term repeats (STRs) from pigmentation genes, we tried to investigate whether genes related to softbiometric attributes can be equally effective.

\subsection{General Approach}

Our theory is that if soft biometric attributes can be used for human classification and identification purposes, then the SNPs related to these soft biometrics may provide useful information in classifying individuals based on their ancestral origin. This theory is supported by the fact that individuals within a given ancestral group often have a similar appearance to one another; such as height, weight, or ear size. Given that our panel is different from previously used panels, our panel can be combined with other panels that are primarily pigment based for further improvement in the results.

\subsection{Main Contribution}

This thesis proposes a new distinct panel of 55 single nucleotide polymorphisms for determining ancestral origin. The 55 SNP panel was then reduced to a 22 SNP and 18 SNP panel that have improved results over the 55 SNP panel. This new approach is producing a high success rate, and has great promise for future progress. We chose to create our own panel instead of evaluating or improving upon other panels found in the past [3][11]. This panel is unique in that all prior research has made use of SNPs related to skin pigmentation since this is considered the most readily identifiable difference between different ancestral groups. Our panel did not include pigmentation genes, but are rather based on genes related to important soft biometrics. Similar to published work [12] we chose to use the four common ancestry groups of African American, Asian, European, and Sub-Saharan African.

\subsection{Thesis Organization}

The thesis is separated into five chapters. The first chapter describes the motivation of the project. Chapter 2 provides background information dealing with the project as well as prior related work. Chapter 3 describes the methodology used in the project. Chapter 4 communicates the results of the experiment and Chapter 5 discusses the conclusions drawn. 


\section{Chapter 2}

\section{Background and Related Work}

Chapter 2 provides background information about the problem as well as related research that has been done by others on the problem.

\subsection{DNA Identification}

Watson and Crick [13] were the pioneers of DNA. They were the first group to identify the structure of DNA as well as discuss the existence of base pairs connecting the helix strands. Their paper completely defines the structure of DNA as we know it today. They did not, however, determine how many locations there are in DNA that we could possibly make use of. The Human Genome Sequencing Consortium [14] provided the next step of information about DNA. This project was a 15 year effort to completely map the human genome. The genome was mapped with $99 \%$ accuracy resulting in 2.85 billion base pair locations. This number is the theoretical limit of SNP locations available for our project to make use of. The problem with this large amount of information is storage, $800 \mathrm{MB}$ to store a genome uncompressed or $4 \mathrm{MB}$ to store a genome removing the largely repetitive portions all sequences have in common.

The idea of determining ancestral origin from DNA was developed right along with the discovery of DNA. Neel [15] was the pioneer in the field when he first proposed the idea that some DNA markers may be unique to one population. Neel was actually researching beneficial mutation rates, which naturally lead to the idea that some of these changes may become unique to a certain group of individuals. Chakraborty [16], while doing similar work, hit upon the observation that a novel location in the DNA that exists in a parent will propagate throughout the population and exist only in that population. This propagation also assumes the allele is not harmful to the offspring in some way. The prior work diverges at this point based on what part of the DNA is used for ancestral classification. 


\subsection{Genes}

Shriver [17] found a few alleles commonly used to determine identification in the standard forensic DNA tests that also show a high percentage of success in determining ancestral origin. FY-null, RB2300, LPL, CKMM, PV92, and DRD2 are the six genes determined to be of useful variance levels in this paper. The advantage of using a gene over a Single Nucleotide Polymorphism (SNPs), a single location in the DNA strand, is demonstrated in this paper. Only six genes were used with a high percentage of correct identification of origin between two groups while using other methods require far larger amounts of information.

\subsection{SNPs}

The most successful case using SNPs used 176 SNPs to correctly identify a person's origin. The SNP case may be capable of selecting between four groups but still leaves the problem of disparity in required information available. Frudakis [1] took the idea of genetic ancestry a step further and produced a product still useful today in determining the ancestral origin of an individual between one of three standard groups; European, Sub-Saharan African, and Asian. He used a multivariate classification system to determine what alleles to use from an initial list of 211 SNPs. The final list of 176 alleles is accurate to approximately $98 \%$ at the worst case.

Wetton [2] used a unique SNP profile that only makes use of SNPs from the Y chromosome. Their theory used the idea that as time passes the Y chromosome undergoes very little mutation and maintains the mutations that do occur. Wetton's work shows promise but has the problem of only identifying paternal lineage for males, removing the ability to identify mixed origins as well as female origins. This work is proof that novel SNP panels can provide useful data.

More recently, Kidd [3] used allele frequencies to prove that a panel of 128 previously identified SNPs of pigmentation genes could be used to identify ancestry. Their paper used principle component analysis (PCA) and Ft analysis to determine the allele frequency in the collective population of individuals they used. While this paper did not add new SNPs or classification methods, they did provide population allele frequencies, which can be used in the 
creation of simulated individuals for testing a new classifier. They also showed that the panel could discriminate between members of a large set of 119 ethnic categories.

Nassir [18] produced the work that [3] based their work off of. This new work sought to improve the initial panel by removing those SNPs with the poorest performance from the original panel. Their work is different in that clustering is used for identification, with each cluster being one of the ancestral groups. Classification rate are handled differently because of this. Each individual has the possibility of belonging to any of the clusters so a single individual can belong to multiple clusters at once. This clustering method is one way to deal with the admixture of individuals problem by saying the person is a mix of all clusters he or she belongs to. Errors are now made up of two numbers; the percentage of individuals who should be in the cluster and are not as well as the individuals who are in the cluster and should not be.

\subsection{STRs}

Graydon [11] made use of 15 STR (short term repeats) locations and a Bayesian classifier. The classifier is simple in that a metric is created that defines the probability of each STR profile existing in each of the ancestral groups. Then any two of the profile probabilities are compared and whichever is larger is taken to be the correct profile. This paper made use of the fact that individuals that look different are easier to classify into different ancestral groups. This work proves that locations previously believed to hold no real value in identification can be used with respect to ancestral classification.

Lowe [19] made use of commonly collected STR information for criminals in the United Kingdom. The system made use of 6 STRs as well as a gender-determining STR. They made use of a Bayesian decision rule for determining ancestry. This method only resulted in correct classification of 52.4\% of individuals, with Middle Eastern and Indian sub-continent individuals causing the system the most problems. They, however, did not make use of those STRs containing the most information on ancestral origin, instead making use of just those STRs collected when processing a criminal.

Court [20] used STRs from the Y-chromosome since those STRs have been shown to have the greatest variance between ancestral groups. They chose to use 6 STRs and 600 individuals to test their system. Their system used a classification tree that made decisions based on binary 
classifications. The system correctly identified $82.3 \%$ of individuals into one of three categories; Caucasian, African, and South Asian.

\section{5. mtDNA}

Egeland [21] provided a foundation for the idea of using simulated data for testing a new classifier. They used principle component analysis similar to many others since this is one of the better methods for finding probabilities in prior data. Instead of using SNPs like most others they used mtDNA (mitochondrial DNA) for ancestral identification. The work, however, produced poor results for classification with only a $66.8 \%$ correct classification rate.

\subsection{Classifiers}

There are a few different methods used to identify what ancestral group to which a person belongs. Shriver [17] used a log-likelihood method producing 87\% accuracy, which, while good, is still worse than the results produced by using maximum likelihood and the custom classifier used by Frudakis. Frudakis [1] created a custom classifier with a 98\% accuracy using a scoring rubric taking into account the relative frequencies of certain allele combinations as well as if the combination is homozygous or heterozygous. Frudakis's classifier used a scoring system, zero to 1 , in $1 / 2$ step increments. An individual was ranked a one if they had a homozygous major allele combination, ranked a one-half if they were heterozygous or ranked zero if homozygous for a minor allele combination. This system may have given good results but does not account for cases where the heterozygous combination is the most common in the population. Multiple groups made use of PCA analysis [3][21], while other groups made use of a mix of different methods; the Bayesian decision rule [11][19], clustering [18], and binary trees [20].

\subsection{Novel SNP Selection}

Kayser [12] looked at the possibility of using DNA to predict adult human hair color and eye color. This paper is along the same line that I chose to go, using a novel, visible characteristic linked to DNA to perform some kind of identification. They also speculated that predicting adult human height from DNA is much more difficult than the two traits they chose to use. They speculate that this difficulty is due to the small effect that each SNP has on height 
along with the large overall number of SNPs related to height. The paper also shows a linkage between hair and eye color with skin pigmentation, another significant visual characteristic that many other papers made use of. 


\section{Chapter 3}

\section{Methodology}

\subsection{Data Sets}

SNP selection was done in a non-traditional way compared to other ancestral identification methods. Instead of using pigmentation SNPs, this project used SNPs that are associated with the height, weight, and the ear size of an individual. These three attributes correspond to important soft biometric features that have become of recent interest in human identification [6][22]. This decision was due to the apparent differences in these soft biometrics between different ancestral groups. We collected an initial pool of SNPs comprising every SNP in Entrez known to be associated with one or more of the three attributes. Table 1 shows a summary of the collected data set.

\section{Table 1: Dataset Summary of the Entrez Dataset.}

(A: Asian, AA: African American, E: European, SSA: Sub-Saharan African)

\begin{tabular}{|l|r|r|r|r|}
\hline & Ear Size & Height & Weight & Total \\
\hline A & 344325 & 12928 & 10866 & 368119 \\
\hline AA & 41749 & 1456 & 1308 & 44513 \\
\hline E & 348236 & 12248 & 10520 & 371004 \\
\hline SSA & 292336 & 10306 & 8630 & 311272 \\
\hline Total & 1026646 & 36938 & 31324 & 1094908 \\
\hline
\end{tabular}

\subsection{SNP Profile}

Figure 1 shows the frequency of possible base pair combinations for 3 SNPs. The figure shows that some combinations occur more commonly in nature. This is partially responsible for the incorrect classifications that occurred early in testing. The classifier uses a summation of probabilities for each SNP in the panel. Thus, one group only having a single base pair for that SNP will outweigh any other group that contains 2 or more base pairs for that SNP. This causes 
less of an issue with the current classifier due the summation being done across all SNPs as opposed to using a rank based system at the SNP level. The SNP profile for each ancestral group was created from the Entrez data using the frequency chart described above; assuming no correlation between the separate SNPs. Figure 1 shows example profiles for three SNPs.


Figure 1: Frequency profile for the four ancestry groups for 3 SNPs. (a) rs2518881 (ear size) (b) rs29941 (weight) (c) rs849141 (height). The following notations are used: AAAfrican American; A-Asian; E-European; SSA-Sub-Saharan African 


\subsection{SNP Selection}

For SNP selection, we aimed to identify those SNPs that are most discriminative and distinct for ancestral identification. We tested two methods to determine what SNPs to use in our panel. The two methods tested were using the Euclidian distance between SNP profiles for each group and using the correlation between different SNPs to remove those that were similar.

We used the Pearson correlation coefficient to find the correlation between separate SNP profiles for the same ancestral group. The correlation scores were then added together to get a total correlation score between -4 and +4 for each SNP.

$$
\rho=\frac{\sum_{i=1}^{n}\left(x_{i}-\bar{x}\right)\left(y_{i}-\bar{y}\right)}{(n-1) * \sigma_{x} \sigma_{y}}
$$

This equation represents the correlation between two SNP profiles. $\bar{x}$ and $\bar{y}$ are the respective means of the two distributions being compared and $\sigma_{x}$ and $\sigma_{y}$ are the standard deviations of the distributions. The correlation method provided a panel of three SNPs that had low correlation with all other SNPs. The problem with this method is the panel created was too narrow to provide positive results during testing. A change was made to the correlation to make the correlation score be an average of the correlation score of each SNP for all four ancestral groups instead of a sum of the four separate correlation scores.

We computed the Euclidian distance between different groups for each SNP found. The Euclidian distance was found by using a 16 dimensional vector that corresponded to the possible base-pair combinations for each SNP. The value at each location was the probability for that base-pair combination from the ancestral profiles. The Euclidian distance was done between each of the ancestral groups for each SNP. This resulted in six separate sets of scores, one for each combination of 2 ancestral groups. The distances were found for each of the SNPs in the profile using the following equation.

$$
d_{K, L, S}=\sqrt{\sum_{i=1}^{n}\left(x_{K, i}-x_{L, i}\right)^{2}}
$$


The value of i refers to which of base pair to use from the parent profile at that SNP while $\mathrm{S}$ refers to which SNP the distance measure is for. The subscript K and L refer to two of the ancestral groups. The distances are then used to select what SNPs to use. If the Euclidian distance for a given SNP was above a threshold of 1.12 then the SNP was added to the list of possible SNPs. The value of 1.12 was empirically selected through observation of the square of the Euclidian distances as well as limiting the number of SNPs in the initial panel to a reasonable amount.

The initial panel of SNPs contained 110 entries. Testing was initially done with the panel but results were not very good so we refined the panel. This panel was reduced by removing SNPs that caused problems for people of European ancestry since this group was shown to have the most classification errors using the initial panel. The final modified SNP panel contained the 55 SNPs that provided the best results, 2 SNPs from weight, 6 SNPs from height, and 47 SNPs from ear size. These were distributed over 20 different genes. Table 2 shows the identified panel of 55 SNPs. Impact analysis was used to further reduce this panel to 18 SNPs.

Combining the average Euclidian distance with the average correlation for each SNP provides a graph that can be used to create another possible SNP panel for testing (see Figure 2). The results from this graph are those SNPs that have a small correlation with a large distance. The distance-correlation figure provided a list of possible SNPs that were cross-referenced with the list from the distance method, creating a possible new panel of SNPs to test. Figure 2 shows a scatter plot of the SNP distances and the SNP correlations.

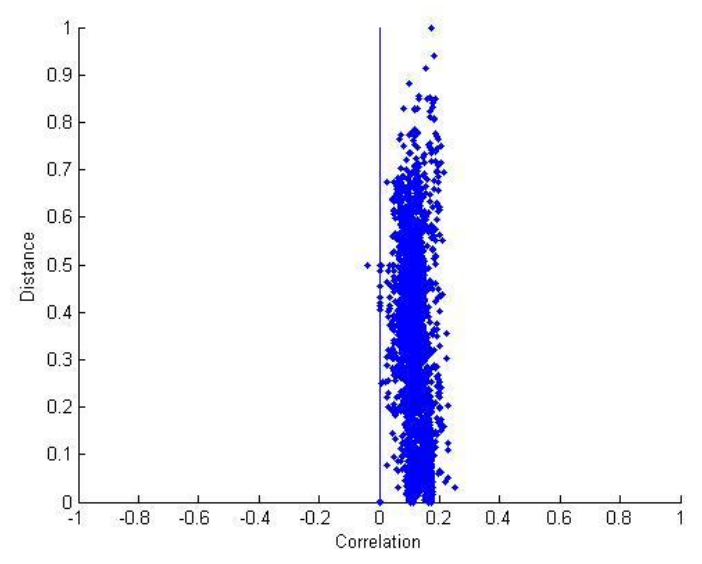

Figure 2: Distance versus correlation plot used for possible SNP panel selection. 
Table 2: Panel of 55 SNPs used in this project.

\begin{tabular}{|c|c|c|c|}
\hline & SNP & Gene & Attribute \\
\hline 1 & rs26797 & ATP2B2 & ear size \\
\hline 2 & rs39640 & ATP2B2 & ear size \\
\hline 3 & rs918244 & ATP2B2 & ear size \\
\hline 4 & rs990397 & ATP2B2 & ear size \\
\hline 5 & rs1473183 & ATP2B2 & ear size \\
\hline 6 & rs17787095 & ATP2B2 & ear size \\
\hline 7 & rs4987850 & BCL2 & ear size \\
\hline 8 & rs12458289 & BCL2 & ear size \\
\hline 9 & rs16917237 & BDNF & ear size \\
\hline 10 & rs1490388 & C6orf173 & Height \\
\hline 11 & rs213938 & CFTR & ear size \\
\hline 12 & rs2283054 & CFTR & ear size \\
\hline 13 & rs2518881 & CFTR & ear size \\
\hline 14 & rs17139943 & CFTR & ear size \\
\hline 15 & rs1337191 & COL11A1 & ear size \\
\hline 16 & rs1415364 & COL11A1 & ear size \\
\hline 17 & rs2061705 & COL11A1 & ear size \\
\hline 18 & rs2376280 & COL11A1 & ear size \\
\hline 19 & rs2376281 & COL11A1 & ear size \\
\hline 20 & rs4907986 & COL11A1 & ear size \\
\hline 21 & rs4908286 & COL11A1 & ear size \\
\hline 22 & rs4908287 & COL11A1 & ear size \\
\hline 23 & rs4908288 & COL11A1 & ear size \\
\hline 24 & rs4908290 & COL11A1 & ear size \\
\hline 25 & rs4908291 & COL11A1 & ear size \\
\hline 26 & rs7517682 & COL11A1 & ear size \\
\hline 27 & rs9970114 & COL11A1 & ear size \\
\hline 28 & rs10874679 & COL11A1 & ear size \\
\hline 29 & rs11164636 & COL11A1 & ear size \\
\hline
\end{tabular}

\begin{tabular}{|c|c|c|c|}
\hline 30 & rs11164653 & COL11A1 & ear size \\
\hline 31 & rs11164665 & COL11A1 & ear size \\
\hline 32 & rs11164668 & COL11A1 & ear size \\
\hline 33 & rs11164669 & COL11A1 & ear size \\
\hline 34 & rs11164672 & COL11A1 & ear size \\
\hline 35 & rs11164673 & COL11A1 & ear size \\
\hline 36 & rs12042830 & COL11A1 & ear size \\
\hline 37 & rs213944 & CTFR & ear size \\
\hline 38 & rs213952 & CTFR & ear size \\
\hline 39 & rs3791679 & EFEMP1 & Height \\
\hline 40 & rs1860131 & EFG & ear size \\
\hline 41 & rs2237048 & EGF & ear size \\
\hline 42 & rs488133 & ESR1 & ear size \\
\hline 43 & rs3135761 & FGFR2 & ear size \\
\hline 44 & rs4752566 & FGFR2 & ear size \\
\hline 45 & rs4896582 & GPR126 & Height \\
\hline 46 & rs10946808 & HIST1H1D & Height \\
\hline 47 & rs2288377 & IGF1 & ear size \\
\hline 48 & rs5742637 & IGF1 & ear size \\
\hline 49 & rs5742639 & IGF1 & ear size \\
\hline 50 & rs1520223 & IGF1 & Height \\
\hline 51 & rs11895564 & ITGA6 & ear size \\
\hline 52 & rs849141 & JAZF1 & Height \\
\hline 53 & rs29941 & KCTD15, CHST8 & Weight \\
\hline 54 & rs1973993 & $N R$ & Weight \\
\hline 55 & rs8179181 & TGFB1 & ear size \\
\hline
\end{tabular}


Another selection method was tested that works similarly. The alternative selection method involved using the average Euclidean distance of an SNP with the average of the distances between ancestral groups for a given SNP (see Figure 3). The distance between two ancestral groups for a certain SNP is found by taking the Euclidean distance of the SNP profile for each of those ancestral groups. The SNPs selected from this graph are those closest to the upper right corner, corresponding to those SNPs that have the highest distance between ancestral groups as well as those SNPs that are the least similar to other SNPs in the panel.

The final step of SNP selection was done by performing an impact analysis on the SNP panel. The impact analysis was used to remove the poorest performing SNPs from our panel to reduce the overall computational complexity of the system as well as reducing the amount of error in the system. The knockout analysis removed 37 SNPs from the panel (resulting in 18 SNPs) and dropped the average error from $2.33 \%$ to $0.05 \%$. The final SNP panel is shown in Table 3. The impact analysis completely removed weight-related SNPs from the panel and reduced the number of height-related SNPs to 3 and the number of ear-related SNPs to 15. Figure 4 shows a curve of classification error versus the number of SNPs in the SNP panels. The panel selected corresponds to the least error seen from any of the SNP panels.

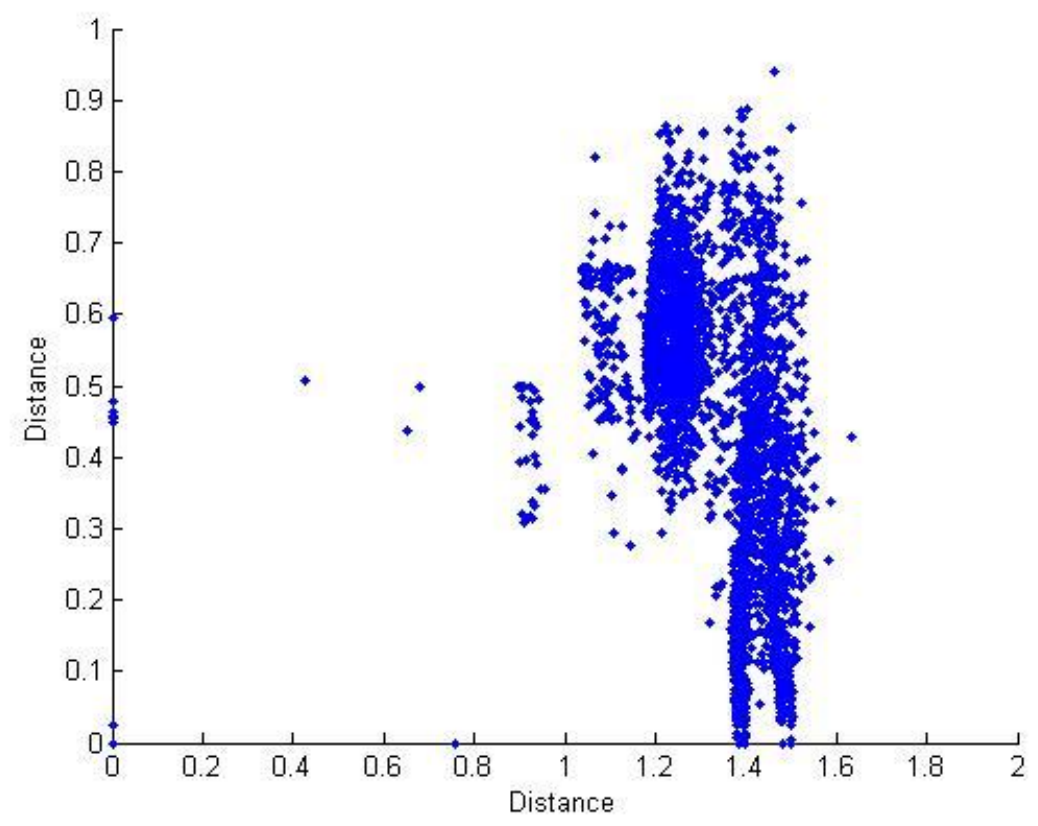

Figure 3: Across SNP distance versus inter-class SNP distance. 
Table 3: Panel of 18 SNPs found after the impact analysis.

\begin{tabular}{|l|l|l|}
\hline SNP & Gene & Attribute \\
\hline rs10946808 & HIST1H1D & Height \\
\hline rs11164636 & COL11A1 & ear size \\
\hline rs11164665 & COL11A1 & ear size \\
\hline rs12042830 & COL11A1 & ear size \\
\hline rs12458289 & BCL2 & ear size \\
\hline rs1337191 & COL11A1 & ear size \\
\hline rs1520223 & IGF1 & Height \\
\hline rs1860131 & EFG & ear size \\
\hline rs2061705 & COL11A1 & ear size \\
\hline rs213944 & CTFR & ear size \\
\hline rs2237048 & EGF & ear size \\
\hline rs2288377 & IGF1 & ear size \\
\hline rs4752566 & FGFR2 & ear size \\
\hline rs4896582 & GPR126 & Height \\
\hline rs4987850 & BCL2 & ear size \\
\hline rs5742637 & IGF1 & ear size \\
\hline rs5742639 & IGF1 & ear size \\
\hline rs990397 & ATP2B2 & ear size \\
\hline
\end{tabular}



Figure 4: Error Curve used to find the SNP Panel with the lowest error. 


\subsection{Classification}

Multiple methods were tested for the classifier to determine which classifier would work best on the data set. Having constructed the feature space, a classifier was then used to separate individuals into different ancestral groups. The classifiers tested included k-nearest neighbor, maximum-likelihood with thresholds and without thresholds, support vector machine, and a fusion of the above three classifiers (maximum-likelihood with thresholds was removed as well as $\mathrm{KNN}$ at $\mathrm{K}=3$ and $\mathrm{KNN}$ at $\mathrm{K}=5$ so the classifiers used would be unique). Classification error in this thesis is determined by taking the average error for all of the ancestral groups when a piecewise comparison was done. Total classification error refers to the sum of the errors for each individual group.

\subsubsection{K-Nearest Neighbor}

The k-nearest neighbor algorithm gave the worst results of all of the classifiers tested. The tests were done using $\mathrm{k}=1,3$, and 5 to discount errors due to an individual being an outlier from his or her ancestral group. The nearest neighbor was found by comparing an individual from the testing data and finding the individual from the training data that matches that individual the closest. When using $\mathrm{k}=3,5$ the most common ancestral group in the k-nearest neighbors is used to classify the individual. Using $\mathrm{k}=3$ or $\mathrm{k}=5$ did not fix the error problems because there were so many outlier individuals in each ancestral group.

\subsubsection{Maximum Likelihood}

The maximum-likelihood classifier worked better than the support vector machine and the k-nearest neighbor classifiers but still has some error. The maximum-likelihood was taken with respect to the four SNP profiles created from the training data set. These results showed some ancestral groups could benefit from a threshold but since all ancestral groups do not benefit the threshold was not used. Given the SNPs from an unknown person, we compute the score for the $k^{\text {th }}$ ancestral group as follows:

$$
\text { score }_{k}=\sum_{i=1}^{n} P\left(S N P_{i} \mid A P_{k}\right)
$$

where $A P_{k}$ is the ancestral profile for group $\mathrm{k}$. 
The maximum-likelihood classifier takes an individual and compares them to each of the four SNP profiles to find which profile the individual is closest to. Each individual was compared to the training profiles and the probability of each base-pair combination that occurred in the testing individual was added together, creating the matching score for that profile. The ancestral group whose profile gave the largest result was chosen as the predicted group:

$$
\text { predicted ancestry group }=\arg \max _{k}\left\{\text { score }_{k}\right\}
$$

Another feature of the maximum-likelihood classifier was that the classifier also could say "no match" was found if the top two scores were too close. The decided score difference was 5 from empirical testing. This helped deal with incorrect classification problems as well as dealing with an individual of mixed ancestral origin. The results shown in this thesis correspond to not having the "no match" case as an option. When the no match case is put into use no error is found in classification of any of the ancestral groups but the "no match" rates are around 90\% for the European ancestral group.

\subsubsection{Support Vector Machine}

The support vector machine (SVM) classifier gave similar results to the nearest neighbor algorithm. The MATLAB SVM was used to perform the SVM tests. The support vector machine used machine learning to make a decision about what class an object belonged to. The labeled training data set is passed to the SVM to define what the different classes are. This SVM used a quadratic plane to separate the data sets as opposed to the least-squares method of the sequential minimal optimization method. A layered argument is needed to classify using an SVM with more than two classes. A decision tree was used to handle having more than two classes. Initially the data set was labeled as Asians or others. If the individual belonged to the Asian group then classification is performed, otherwise the next tier in the decision tree is reached. The individuals not classified as Asian are then classified as African Americans or others. The same decisions were made for each tier in the decision tree. Tier 3 separated the Europeans from the Sub-Saharan Africans. Figure 5 shows the process used. 


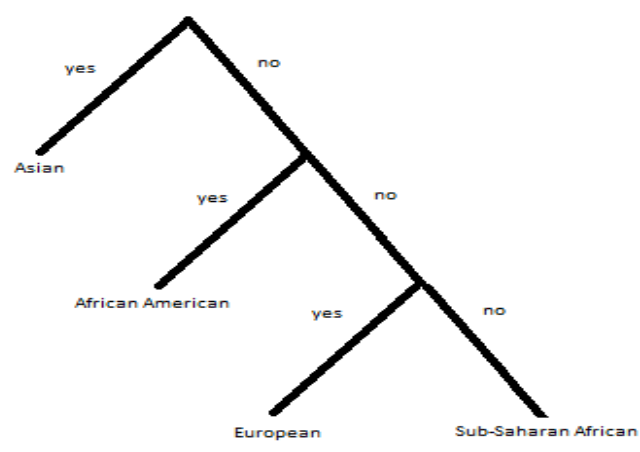

Figure 5: Using the SVM decision tree for multi-group classification.

\subsubsection{Fusion Classifier}

The fusion classifier operated at the decision level by fusing together the decisions provided by the three other classifiers. The results produced by the fusion classifier were worse than any of the other classifiers. This problem was due to the fusion classifier requiring 2 or more classifier that works well and the only functional classifier was the maximum likelihood classifier. Fusion was done by using a majority vote at the decision level. If no majority was found then the results were reported as no result found. The fusion classifier may work better when used with a different SNP panel. For this reason all classifiers must be retested with each new SNP panel created.

\subsection{Feature Space}

The feature space of the problem is made up of the simulated individuals and the profiles for each ancestral group. The columns correspond to each of the SNPs and the rows are made up of the simulated individuals. The bottom 4 rows of the chart contain the density profile for each ancestral group at each SNP. The profiles represent the probability for each of the base-pair combinations at each SNP locations.

\subsection{Impact Analysis}

Impact analysis was done on the final list of SNPs to determine which of the SNPs contributed the most to correct ancestral classification. The SNPs were ranked from most to least importance based on the classification error when that SNP is not used. Knockout was done for the cases of removing one, two, or three SNPs. 


\section{Chapter 4}

\section{Results}

\subsection{Simulated Data}

The generation of individuals to test the classifier proved challenging. We assumed the SNPs for an individual have no correlation. This allows us to use the data from NCBI to create simulated ancestral profiles for individual creation to test our approach. The NCBI data was split into two separate groups for training and testing the classifier. The training group used $75 \%$ of the data while the remaining $25 \%$ of the data went to the testing group. Profiles for each ancestral group were then created from the separate data sets using the method from SNP selection. The distribution of the data was used to find the probability density for a given SNP for each ancestral group. The SNPs of the synthetic individuals were based on a perturbation of the probability distribution for each SNP in the ancestral profile. Each ancestral profile was used in the creation of 1000 individuals for each ancestral group, leading to 4000 individuals in the testing group and 4000 individuals in the training group. The individuals were created by taking the probability distribution for a SNP and generating a base pair combination based on that density. This is repeated for each of the SNPs in the panel to be tested, creating individuals that have the needed SNP panel.

Training individuals were perturbed at a rate of $10 \%$ to simulate the possibility of mutation in the population. The perturbation was done by randomly assigning a SNP value from all possible base pair combinations $10 \%$ of the time instead of using a SNP from the ancestral profile. If the assumption that each of the genes is uncorrelated holds true, then the simulated individuals are valid test subjects.

Figure 6 contains a summary of the different SNP panels used in this project. 


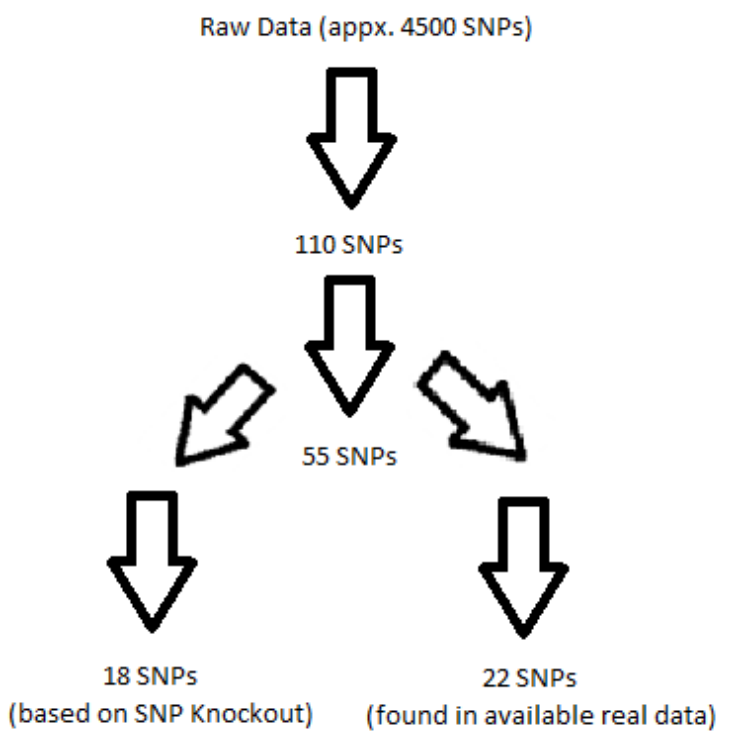

Figure 6: SNP Panels proposed in this work.

The results from using the maximum-likelihood classifier were quite promising for the simulated individuals (see Figure 8 and 9). The chart is read by first looking at the ancestral group listed at the bottom of the chart. Each column corresponds to the percentage of correct classification when only those two groups are used in the classifier, the group listed below that column as well as the label on that column. When discussing an individual of a certain ancestral group this corresponds to the ancestral group listed at the bottom of the chart. No errors occurred when using the classifier on a person of African American, Asian, or Sub-Saharan African Ancestry. Europeans were correctly identified $99.9 \%$ of the time when compared to African Americans. There was a 0.2\% classification error of Europeans being classified as Asians and $0.1 \%$ classification error of Europeans being classified as Sub-Saharan Africans. Considering that some of the earlier results in the area of ancestral classification considered approximately $70 \%$ to be a success [23], then these amounts of error are acceptable as well as better performing than all other research in this area, such as Frudakis at 98\% successful classification. 


\subsubsection{Error Resilience}

The error shown in Figure 7 represents the error the classifier has when used on the simulated data. The error remains level for all but the $100 \%$ perturbation case because perturbation is done by randomly assigning a new base pair combination from all possible base pair combinations. This means that those SNP profiles that have only one base pair combination have a $6.25 \%$ chance of being correct when randomly assigned. This chance goes up for each peak in the SNP profile, meaning groups are more resilient to error if they have multiple peaks. This type of perturbation levels off the peaks in the SNP profile but the peaks will still exist until $100 \%$ perturbation occurs.

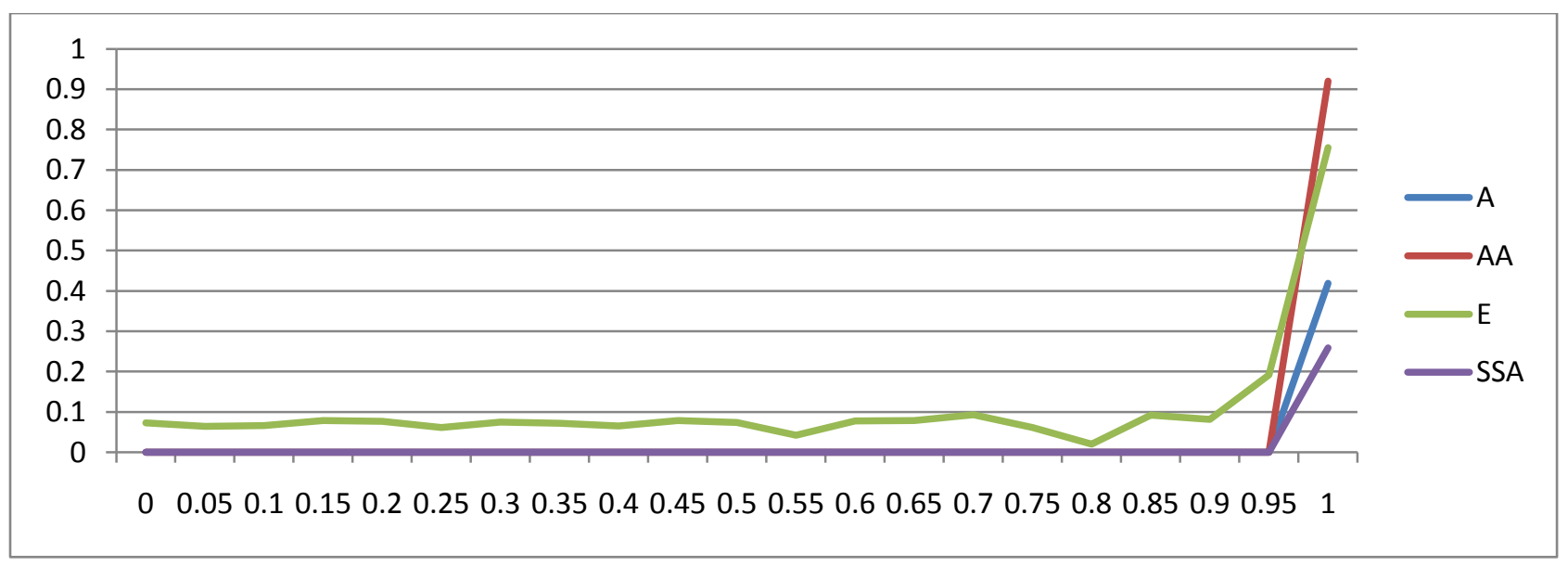

Figure 7: The amount of error for each ancestral group with different perturbation rates.

\subsubsection{Impact Analysis Results}

Tables 4 and 5 summarize the impact analysis results for the simulated data of 55 SNPs. The previous error before the knockout analysis was $0.02(2 \%)$. 
Table 4: Single SNP knockout results, 55 SNPs. (Top 20 SNPs with most impact)

\begin{tabular}{|c|c|c|c|c|c|c|c|}
\hline SNP1 & $\begin{array}{l}\text { SNP1 } \\
\text { Attribute }\end{array}$ & $\begin{array}{l}\text { A } \\
\text { Error }\end{array}$ & $\begin{array}{l}\text { AA } \\
\text { Error }\end{array}$ & $\begin{array}{l}\mathrm{E} \\
\text { Error }\end{array}$ & $\begin{array}{l}\text { SSA } \\
\text { Error }\end{array}$ & $\begin{array}{l}\text { Total } \\
\text { Error }\end{array}$ & $\begin{array}{l}\text { Error } \\
\text { Difference }(\%)\end{array}$ \\
\hline rs11164673 & ear size & 0.0000 & 0.0000 & 0.1580 & 0.0000 & 0.0395 & 97.5000 \\
\hline rs10874679 & ear size & 0.0000 & 0.0000 & 0.1030 & 0.0000 & 0.0258 & 28.7500 \\
\hline rs2376280 & ear size & 0.0000 & 0.0000 & 0.0950 & 0.0000 & 0.0238 & 18.7500 \\
\hline rs488133 & ear size & 0.0000 & 0.0000 & 0.0930 & 0.0000 & 0.0233 & 16.2500 \\
\hline rs1490388 & Height & 0.0000 & 0.0000 & 0.0920 & 0.0000 & 0.0230 & 15.0000 \\
\hline rs4908288 & ear size & 0.0000 & 0.0000 & 0.0920 & 0.0000 & 0.0230 & 15.0000 \\
\hline rs2283054 & ear size & 0.0000 & 0.0000 & 0.0910 & 0.0000 & 0.0228 & 13.7500 \\
\hline rs4907986 & ear size & 0.0000 & 0.0000 & 0.0900 & 0.0000 & 0.0225 & 12.5000 \\
\hline rs213944 & ear size & 0.0000 & 0.0000 & 0.0900 & 0.0000 & 0.0225 & 12.5000 \\
\hline rs2288377 & ear size & 0.0000 & 0.0000 & 0.0900 & 0.0000 & 0.0225 & 12.5000 \\
\hline rs5742637 & ear size & 0.0000 & 0.0000 & 0.0900 & 0.0000 & 0.0225 & 12.5000 \\
\hline rs39640 & ear size & 0.0000 & 0.0000 & 0.0890 & 0.0000 & 0.0223 & 11.2500 \\
\hline rs9970114 & ear size & 0.0000 & 0.0000 & 0.0890 & 0.0000 & 0.0223 & 11.2500 \\
\hline rs849141 & Height & 0.0000 & 0.0000 & 0.0890 & 0.0000 & 0.0223 & 11.2500 \\
\hline rs 16917237 & ear size & 0.0000 & 0.0000 & 0.0880 & 0.0000 & 0.0220 & 10.0000 \\
\hline rs17139943 & ear size & 0.0000 & 0.0000 & 0.0880 & 0.0000 & 0.0220 & 10.0000 \\
\hline rs7517682 & ear size & 0.0000 & 0.0000 & 0.0880 & 0.0000 & 0.0220 & 10.0000 \\
\hline rs4896582 & Height & 0.0000 & 0.0000 & 0.0880 & 0.0000 & 0.0220 & 10.0000 \\
\hline rs 17787095 & ear size & 0.0000 & 0.0000 & 0.0860 & 0.0000 & 0.0215 & 7.5000 \\
\hline rs213938 & ear size & 0.0000 & 0.0000 & 0.0860 & 0.0000 & 0.0215 & 7.5000 \\
\hline
\end{tabular}

Table 5 and 6 show that certain SNPs provide more information for classification purposes than other SNPs. Table 5 provides a list of those SNPs that have the highest impact to classification. Table 6 contains the results for what pairs of SNPs provide the most classification information when used together. The SNPs at the top of each list are those that are most important for the classifier to work well. 
Table 5: Double SNP knockout results, 55 SNPs. (Top 20 SNP pairs with most impact)

\begin{tabular}{|c|c|c|c|c|c|c|c|c|c|}
\hline SNP1 & SNP2 & $\begin{array}{l}\text { SNP1 } \\
\text { Attribute }\end{array}$ & $\begin{array}{l}\text { SNP2 } \\
\text { Attribute }\end{array}$ & $\begin{array}{l}\text { A } \\
\text { Error }\end{array}$ & $\begin{array}{l}\text { AA } \\
\text { Error }\end{array}$ & $\begin{array}{l}\mathrm{E} \\
\text { Error }\end{array}$ & $\begin{array}{l}\text { SSA } \\
\text { Error }\end{array}$ & $\begin{array}{l}\text { Total } \\
\text { Error }\end{array}$ & $\begin{array}{l}\text { Error } \\
\text { Difference }(\%)\end{array}$ \\
\hline rs4896582 & rs4987850 & ear size & ear size & 0.0000 & 0.0000 & 0.2040 & 0.0000 & 0.0510 & 155.0000 \\
\hline rs2061705 & rs4987850 & ear size & ear size & 0.0000 & 0.0000 & 0.1870 & 0.0000 & 0.0468 & 133.7500 \\
\hline rs4987850 & rs10946808 & ear size & ear size & 0.0000 & 0.0000 & 0.1810 & 0.0000 & 0.0453 & 126.2500 \\
\hline rs4908287 & rs4987850 & ear size & ear size & 0.0000 & 0.0000 & 0.1790 & 0.0000 & 0.0448 & 123.7500 \\
\hline rs2376281 & rs4987850 & ear size & ear size & 0.0000 & 0.0000 & 0.1780 & 0.0000 & 0.0445 & 122.5000 \\
\hline rs2237048 & rs4987850 & ear size & ear size & 0.0000 & 0.0000 & 0.1770 & 0.0000 & 0.0443 & 121.2500 \\
\hline rs4987850 & rs11164669 & ear size & ear size & 0.0000 & 0.0000 & 0.1760 & 0.0000 & 0.0440 & 120.0000 \\
\hline rs4987850 & rs5742639 & ear size & ear size & 0.0000 & 0.0000 & 0.1750 & 0.0000 & 0.0438 & 118.7500 \\
\hline rs4987850 & rs11164665 & ear size & Height & 0.0000 & 0.0000 & 0.1750 & 0.0000 & 0.0438 & 118.7500 \\
\hline rs 1520223 & rs4987850 & ear size & ear size & 0.0000 & 0.0000 & 0.1740 & 0.0000 & 0.0435 & 117.5000 \\
\hline rs2283054 & rs4987850 & ear size & ear size & 0.0000 & 0.0000 & 0.1730 & 0.0000 & 0.0433 & 116.2500 \\
\hline rs4987850 & rs5742637 & ear size & ear size & 0.0000 & 0.0000 & 0.1730 & 0.0000 & 0.0433 & 116.2500 \\
\hline rs4987850 & rs11164673 & ear size & ear size & 0.0000 & 0.0000 & 0.1720 & 0.0000 & 0.0430 & 115.0000 \\
\hline rs29941 & rs4987850 & ear size & ear size & 0.0000 & 0.0000 & 0.1710 & 0.0000 & 0.0428 & 113.7500 \\
\hline rs918244 & rs4987850 & ear size & ear size & 0.0000 & 0.0000 & 0.1710 & 0.0000 & 0.0428 & 113.7500 \\
\hline rs2288377 & rs4987850 & ear size & ear size & 0.0000 & 0.0000 & 0.1710 & 0.0000 & 0.0428 & 113.7500 \\
\hline rs4987850 & rs9970114 & ear size & ear size & 0.0000 & 0.0000 & 0.1710 & 0.0000 & 0.0428 & 113.7500 \\
\hline rs990397 & rs4987850 & Height & ear size & 0.0000 & 0.0000 & 0.1700 & 0.0000 & 0.0425 & 112.5000 \\
\hline rs1415364 & rs4987850 & ear size & ear size & 0.0000 & 0.0000 & 0.1700 & 0.0000 & 0.0425 & 112.5000 \\
\hline rs 1860131 & rs4987850 & ear size & ear size & 0.0000 & 0.0000 & 0.1700 & 0.0000 & 0.0425 & 112.5000 \\
\hline
\end{tabular}

\subsection{Real Data}

The individuals in the HGDP data set [24] were used to test classifier functionality. The HGDP data set has 890 individuals usable for testing the classifier; 462 Asians, 327 Europeans, and 101 Sub-Saharan Africans. The 890 individuals out of the 1042 were those that the ancestral group was available and fell into one of our four ancestral groups. The complete data set could not be used due to some individuals not belonging to any of the four groups we considered and individuals being marked as not complete by the collection group. Of the 55 SNPs proposed, only 22 SNPs were found in the HGDP data set. Thus, we restricted our analysis to a panel of those 22 SNPs. Table 6 shows the reduced 22 SNP panel. Testing was done using the available data from the HGDP data set. The testing was done using the reduced data set under the 
assumption the available SNP panel would still provide enough information for correct classification. This increased the error from $2 \%$ to $14 \%$. This increase in error was unforeseen but was still under the amount of error other papers deemed acceptable [23]. Another problem with the data set was that it did not include African American, thus removing one of the groups the classifier was trained on. Due to this fact no testing was done to verify success rates of African American classification using real data.

Table 6: Panel of 22 SNPs used on real data.

\begin{tabular}{|l|l|l|}
\hline SNP & Gene & Attribute \\
\hline rs26797 & ATP2B2 & ear size \\
\hline rs4987850 & BCL2 & ear size \\
\hline rs12458289 & BCL2 & ear size \\
\hline rs1490388 & C6orf173 & height \\
\hline rs213938 & CFTR & ear size \\
\hline rs2283054 & CFTR & ear size \\
\hline rs2061705 & COL11A1 & ear size \\
\hline rs2376280 & COL11A1 & ear size \\
\hline rs4908290 & COL11A1 & ear size \\
\hline rs11164665 & COL11A1 & ear size \\
\hline rs213952 & CTFR & ear size \\
\hline rs3791679 & EFEMP1 & height \\
\hline rs488133 & ESR1 & ear size \\
\hline rs3135761 & FGFR2 & ear size \\
\hline rs4752566 & FGFR2 & ear size \\
\hline rs10946808 & HIST1H1D & height \\
\hline rs5742637 & IGF1 & ear size \\
\hline rs11895564 & ITGA6 & ear size \\
\hline rs849141 & JAZF1 & height \\
\hline rs29941 & KCTD15, CHST8 & weight \\
\hline rs1973993 & NR & weight \\
\hline rs8179181 & TGFB1 & ear size \\
\hline
\end{tabular}


We mentioned that, for the 18-SNP panel (produced after knock-out experiments on the 55-SNP panel) which produced an error of 0.02 on the simulated data, only 7 of the 18 SNPs were found in individuals in the HGDP data set. These 7 SNPs were not enough to discriminate between the ancestral groups in the data set. Thus, the 18-SNP panel could not be tested on real data.

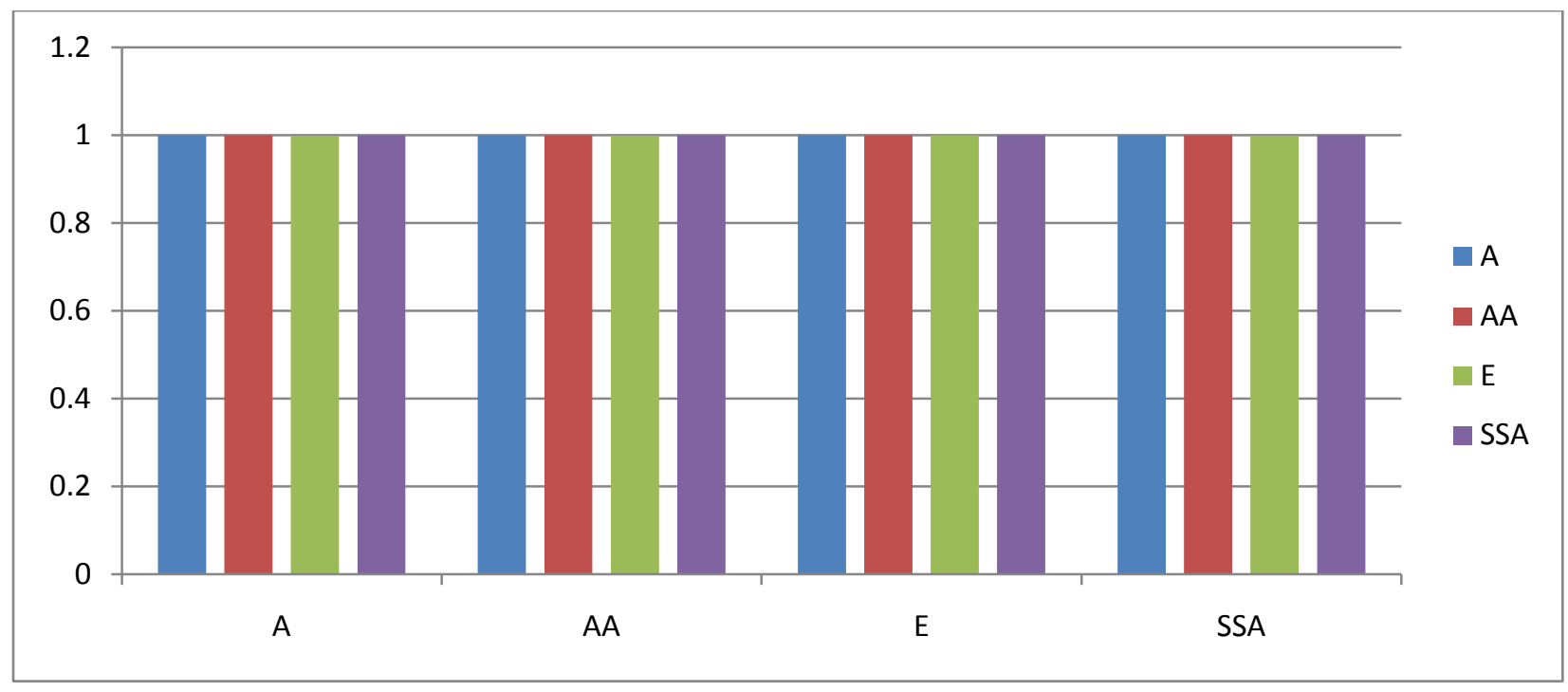

Figure 8: Results of pair-wise comparisons between different ancestry groups using the proposed panel of 18 SNPs (simulated data).

The pair-wise comparison of the panel of 18 SNPs showed that in the theoretical case the classifier worked extremely well with almost no error. Only 7 SNPs of the 18 SNP panel were found in the real data set so the panel of 22 SNPs, those available from the 55 SNP panel, were used to show that the classifier produces comparable results on real data. The panel of 7 SNPs could not be used because all of the SNPs available in that panel were low on the impact analysis, meaning the SNPs were those that contribute little to the classifier accuracy. 


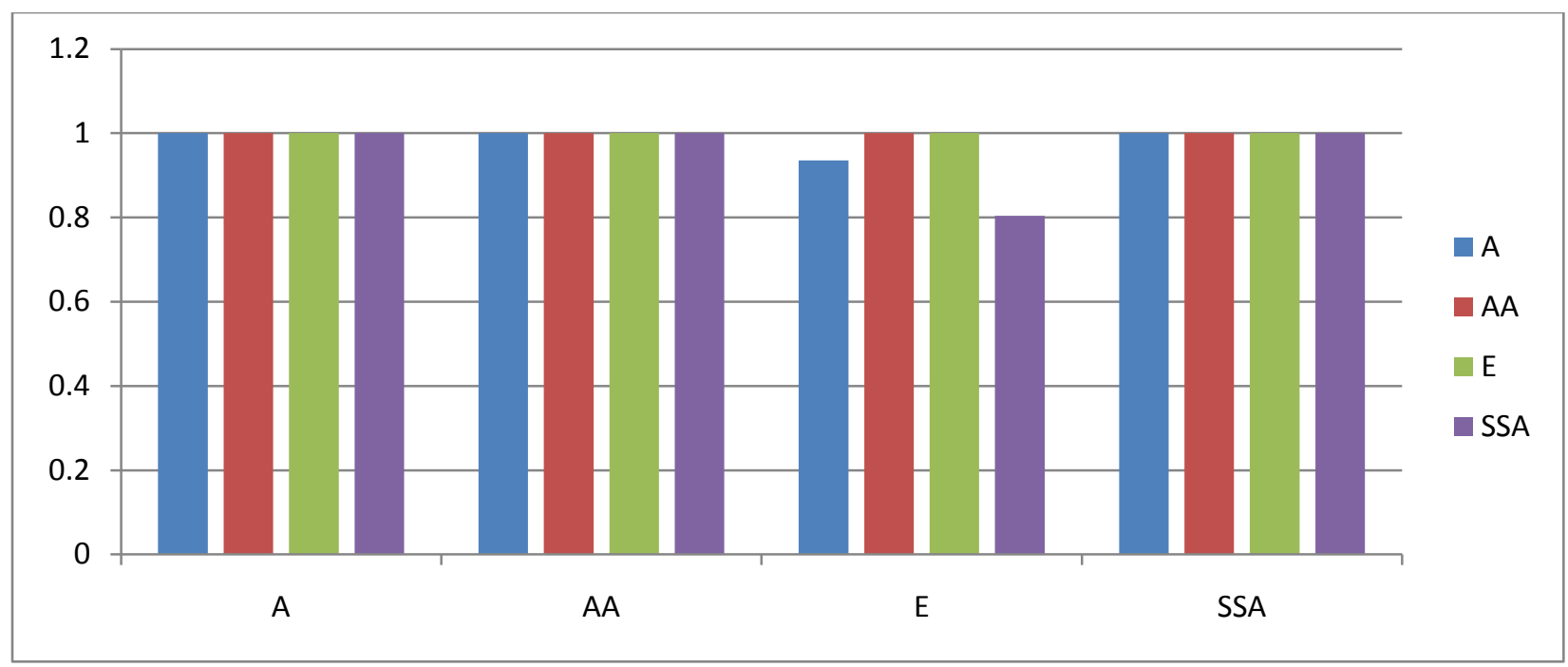

Figure 9: Results of pair-wise comparisons between different ancestry groups using the proposed panel of 22 SNPs (simulated data).

The results from using a maximum-likelihood classifier are also quite promising for the HGDP data set (see Figure 10). No errors occurred when using the classifier on a person of SubSaharan African Ancestry. Europeans were correctly identified 70\% of the time when compared to Asians and $68 \%$ of the time when compared to Sub-Saharan Africans. Asians were correctly identified $84 \%$ of the time when classified with Europeans and $83 \%$ of the time when classified with Sub-Saharan Africans. The pair-wise error calculations can also be used to estimate the overall error the classifier saw. Using the worst case scenario mentality leads to the conclusion that the worst error rate the classifier could have seen was the error rate of the worst pair-wise comparison. Considering that some earlier work in the area of classification considered approximately $70 \%$ to be success, then these amounts of error are acceptable for all but the European case. The increase in error of the real data over the simulated data is due to limiting the SNP panel from the initial panel of 55 SNPs down to the 22 SNPs available in the HGDP data set. The comparison between Figure 9 and Figure 10 shows that the real data performs poorly in comparison to the simulated data. This error is to be expected due to the relatively small sample sizes available from the HGDP database. 




Figure 10: Results of pair-wise comparisons between different ancestry groups using real data, based on 22 of the 55 SNPs.

\subsubsection{Impact Analysis Results}

Tables 7-9 summarize the impact analysis results for the real data of 22 SNPs. The previous error before the knockout analysis was $0.14(14.0 \%)$.

Table 7: Single SNP knockout results, 22 SNPs (Top 20 SNPs with most impact).

\begin{tabular}{|c|c|c|c|c|c|c|}
\hline SNP & $\begin{array}{l}\text { SNP } \\
\text { Attribute }\end{array}$ & $\begin{array}{l}\text { A } \\
\text { Error }\end{array}$ & $\begin{array}{l}\mathrm{E} \\
\text { Error }\end{array}$ & $\begin{array}{l}\text { SSA } \\
\text { Error }\end{array}$ & $\begin{array}{l}\text { Total } \\
\text { Error }\end{array}$ & $\begin{array}{l}\text { Error } \\
\text { Difference }(\%)\end{array}$ \\
\hline rs849141 & height & 0.2232 & 0.5208 & 0.0588 & 0.2676 & 47.6831 \\
\hline rs11164665 & ear size & 0.1786 & 0.5625 & 0.0588 & 0.2666 & 47.4869 \\
\hline rs12458289 & ear size & 0.2143 & 0.5104 & 0.0588 & 0.2612 & 46.4012 \\
\hline rs 1490388 & height & 0.2054 & 0.5104 & 0.0588 & 0.2582 & 45.7785 \\
\hline rs2061705 & ear size & 0.2143 & 0.4896 & 0.0588 & 0.2542 & 44.9253 \\
\hline rs3135761 & ear size & 0.2232 & 0.4792 & 0.0588 & 0.2537 & 44.8167 \\
\hline rs8179181 & ear size & 0.1964 & 0.4792 & 0.0588 & 0.2448 & 42.8105 \\
\hline rs4987850 & ear size & 0.2143 & 0.5104 & 0.0000 & 0.2416 & 42.0530 \\
\hline rs4908290 & ear size & 0.2232 & 0.4792 & 0.0000 & 0.2341 & 40.1965 \\
\hline rs213952 & ear size & 0.2232 & 0.4792 & 0.0000 & 0.2341 & 40.1965 \\
\hline rs3791679 & height & 0.2232 & 0.4792 & 0.0000 & 0.2341 & 40.1965 \\
\hline rs10946808 & height & 0.2232 & 0.4792 & 0.0000 & 0.2341 & 40.1965 \\
\hline rs5742637 & ear size & 0.2232 & 0.4792 & 0.0000 & 0.2341 & 40.1965 \\
\hline rs29941 & weight & 0.2232 & 0.4792 & 0.0000 & 0.2341 & 40.1965 \\
\hline rs11895564 & ear size & 0.2321 & 0.4583 & 0.0000 & 0.2302 & 39.1833 \\
\hline rs4752566 & ear size & 0.2232 & 0.4583 & 0.0000 & 0.2272 & 38.3803 \\
\hline rs26797 & ear size & 0.2321 & 0.4479 & 0.0000 & 0.2267 & 38.2444 \\
\hline rs2283054 & ear size & 0.2411 & 0.4375 & 0.0000 & 0.2262 & 38.1079 \\
\hline rs 2376280 & ear size & 0.2054 & 0.4688 & 0.0000 & 0.2247 & 37.6947 \\
\hline rs1973993 & weight & 0.2232 & 0.4479 & 0.0000 & 0.2237 & 37.4162 \\
\hline
\end{tabular}


Tables 7-9 show the results of the impact analysis. Table 8 and 9 contain the results for the knockout when a pair or triple of SNPs are removed from the panel all at the same time.

Table 8: Double SNP knockout results, 22 SNPs (Top 20 SNP pairs with most impact).

\begin{tabular}{|c|c|c|c|c|c|c|c|c|}
\hline SNP1 & SNP2 & $\begin{array}{l}\text { SNP1 } \\
\text { Attribute }\end{array}$ & $\begin{array}{l}\text { SNP2 } \\
\text { Attribute }\end{array}$ & $\begin{array}{l}\text { A } \\
\text { Error }\end{array}$ & $\begin{array}{l}\mathrm{E} \\
\text { Error }\end{array}$ & $\begin{array}{l}\text { SSA } \\
\text { Error }\end{array}$ & $\begin{array}{l}\text { Total } \\
\text { Error }\end{array}$ & $\begin{array}{l}\text { Error } \\
\text { Difference }(\%)\end{array}$ \\
\hline rs2061705 & rs11164665 & ear size & ear size & 0.2054 & 0.5938 & 0.0588 & 0.286 & 51.048951 \\
\hline rs12458289 & rs11164665 & ear size & ear size & 0.1875 & 0.5833 & 0.0588 & 0.2766 & 49.385394 \\
\hline rs1490388 & rs11164665 & height & ear size & 0.1875 & 0.5729 & 0.0588 & 0.2731 & 48.736726 \\
\hline rs26797 & rs11164665 & ear size & ear size & 0.1964 & 0.5625 & 0.0588 & 0.2726 & 48.6427 \\
\hline rs11164665 & rs3135761 & ear size & ear size & 0.2143 & 0.5417 & 0.0588 & 0.2716 & 48.453608 \\
\hline rs4987850 & rs849141 & ear size & height & 0.2232 & 0.5313 & 0.0588 & 0.2711 & 48.358539 \\
\hline rs 12458289 & rs1490388 & ear size & height & 0.2232 & 0.5313 & 0.0588 & 0.2711 & 48.358539 \\
\hline rs 12458289 & rs849141 & ear size & height & 0.2232 & 0.5313 & 0.0588 & 0.2711 & 48.358539 \\
\hline rs2061705 & rs 2376280 & ear size & ear size & 0.2054 & 0.4896 & 0.1176 & 0.2709 & 48.320413 \\
\hline rs4987850 & rs11164665 & ear size & ear size & 0.1786 & 0.5729 & 0.0588 & 0.2701 & 48.167345 \\
\hline rs213938 & rs2061705 & ear size & ear size & 0.2321 & 0.4583 & 0.1176 & 0.2694 & 48.032665 \\
\hline rs4987850 & rs2061705 & ear size & ear size & 0.2232 & 0.5208 & 0.0588 & 0.2676 & 47.683109 \\
\hline rs3791679 & rs849141 & height & height & 0.2232 & 0.5208 & 0.0588 & 0.2676 & 47.683109 \\
\hline rs10946808 & rs849141 & height & height & 0.2232 & 0.5208 & 0.0588 & 0.2676 & 47.683109 \\
\hline rs 2376280 & rs11164665 & ear size & ear size & 0.1786 & 0.5625 & 0.0588 & 0.2666 & 47.486872 \\
\hline rs11164665 & rs3791679 & ear size & height & 0.1786 & 0.5625 & 0.0588 & 0.2666 & 47.486872 \\
\hline rs11164665 & rs10946808 & ear size & height & 0.1786 & 0.5625 & 0.0588 & 0.2666 & 47.486872 \\
\hline rs11164665 & rs5742637 & ear size & ear size & 0.1786 & 0.5625 & 0.0588 & 0.2666 & 47.486872 \\
\hline rs5742637 & rs849141 & ear size & height & 0.2143 & 0.5208 & 0.0588 & 0.2646 & 47.089947 \\
\hline rs12458289 & rs2061705 & ear size & ear size & 0.2232 & 0.5104 & 0.0588 & 0.2642 & 47.009841 \\
\hline
\end{tabular}


Table 9: Triple SNP knockout results, 22 SNPs (Top 20 SNP triplets with most impact).

\begin{tabular}{|c|c|c|c|c|c|c|c|c|c|c|}
\hline SNP1 & SNP2 & SNP3 & $\begin{array}{l}\text { SNP1 } \\
\text { Attribute }\end{array}$ & $\begin{array}{l}\text { SNP2 } \\
\text { Attribute }\end{array}$ & $\begin{array}{l}\text { SNP2 } \\
\text { Attribute }\end{array}$ & $\begin{array}{l}\text { A } \\
\text { Error }\end{array}$ & $\begin{array}{l}\mathrm{E} \\
\text { Error }\end{array}$ & $\begin{array}{l}\text { SSA } \\
\text { Error }\end{array}$ & $\begin{array}{l}\text { Total } \\
\text { Error }\end{array}$ & $\begin{array}{l}\text { Error } \\
\text { Difference }(\%)\end{array}$ \\
\hline rs1490388 & rs1973993 & rs2283054 & ear size & ear size & ear size & 0.1875 & 0.6042 & 0.1176 & 0.3031 & 53.810624 \\
\hline rs488133 & rs1490388 & rs2283054 & ear size & ear size & ear size & 0.2321 & 0.5521 & 0.1176 & 0.3006 & 53.42648 \\
\hline rs213938 & rs213952 & rs2376280 & ear size & Height & ear size & 0.25 & 0.5833 & 0.0588 & 0.2974 & 52.925353 \\
\hline rs213938 & rs213952 & rs11164665 & ear size & Height & weight & 0.25 & 0.5833 & 0.0588 & 0.2974 & 52.925353 \\
\hline rs213938 & rs1490388 & rs 2283054 & ear size & ear size & ear size & 0.2054 & 0.625 & 0.0588 & 0.2964 & 52.766532 \\
\hline rs213938 & rs213952 & rs2283054 & ear size & Height & ear size & 0.2054 & 0.6146 & 0.0588 & 0.2929 & 52.202117 \\
\hline rs26797 & rs1490388 & rs2283054 & ear size & ear size & ear size & 0.2321 & 0.5833 & 0.0588 & 0.2914 & 51.956074 \\
\hline rs1490388 & rs2283054 & rs3791679 & ear size & ear size & ear size & 0.1875 & 0.6146 & 0.0588 & 0.287 & 51.219512 \\
\hline rs1490388 & rs2283054 & rs3135761 & ear size & ear size & height & 0.2054 & 0.5938 & 0.0588 & 0.286 & 51.048951 \\
\hline rs1490388 & rs2283054 & rs4987850 & ear size & ear size & height & 0.2054 & 0.5938 & 0.0588 & 0.286 & 51.048951 \\
\hline rs2061705 & rs11164665 & rs5742637 & ear size & ear size & ear size & 0.2054 & 0.5938 & 0.0588 & 0.286 & 51.048951 \\
\hline rs2061705 & rs11164665 & rs 3135761 & ear size & ear size & ear size & 0.2232 & 0.5729 & 0.0588 & 0.285 & 50.877193 \\
\hline rs26797 & rs11164665 & rs849141 & ear size & ear size & height & 0.2054 & 0.5833 & 0.0588 & 0.2825 & 50.442478 \\
\hline rs2061705 & rs2376280 & rs3135761 & ear size & ear size & ear size & 0.25 & 0.4792 & 0.1176 & 0.2823 & 50.407368 \\
\hline rs11164665 & rs3135761 & rs849141 & ear size & ear size & height & 0.2232 & 0.5625 & 0.0588 & 0.2815 & 50.26643 \\
\hline rs213938 & rs 2376280 & rs11164665 & ear size & ear size & ear size & 0.2143 & 0.5104 & 0.1176 & 0.2808 & 50.14245 \\
\hline rs26797 & rs11164665 & rs3135761 & ear size & ear size & ear size & 0.2411 & 0.5417 & 0.0588 & 0.2805 & 50.089127 \\
\hline rs4987850 & rs2061705 & rs 2376280 & ear size & ear size & ear size & 0.2411 & 0.5417 & 0.0588 & 0.2805 & 50.089127 \\
\hline rs12458289 & rs1490388 & rs3135761 & ear size & Height & ear size & 0.2411 & 0.5417 & 0.0588 & 0.2805 & 50.089127 \\
\hline rs1490388 & rs 2061705 & rs11164665 & Height & ear size & ear size & 0.1875 & 0.5938 & 0.0588 & 0.28 & 50 \\
\hline
\end{tabular}

\subsection{Comparative Results}

Table 10 contains a summary of the results for each of the papers referenced by this project. Kidd [3] is omitted from the table because they did not specifically discuss results other than to say the results were good. Our method when used on real data has comparable results to the majority of the other groups. While [1] has better results they make use of nearly 10 times the amount of information that our panel makes use of and [17] uses genes, which contain much more information than an individual SNP is capable of. 
Table 10: Comparative results of the different methods described in this project.

\begin{tabular}{|l|l|r|l|r|l|}
\hline Method & Type of Data & Size & Real/Simulated & Classification Rate & Reference \\
\hline Log-likelihood & Genes & 565 & Real & 0.994 & {$[17]$} \\
\hline Bayesian & STR & 23186 & Real & 0.9 & {$[11]$} \\
\hline PCA & mtDNA & 2100 & Simulated & 0.781 & {$[21]$} \\
\hline PCA & mtDNA & 2017 & Real & 0.668 & {$[21]$} \\
\hline Multivariate & SNP & 230 & Real & 0.98 & {$[1]$} \\
\hline MFI & Y-Chromosome & 627 & Real & 0.803 & {$[2]$} \\
\hline Ours-55 & SNP & 4000 & Simulated & 0.97 & Ours \\
\hline Ours-22 & SNP & 4000 & Simulated & 0.93 & Ours \\
\hline Ours-22 & SNP & 890 & Real & 0.86 & Ours \\
\hline Ours-18 & SNP & 4000 & Simulated & 0.998 & Ours \\
\hline
\end{tabular}




\section{Chapter 5}

\section{Conclusions}

\subsection{Conclusions}

This research shows ancestry identification using SNPs related to body build works as well as classification done using the more traditional pigmentation SNPs. The main outcome of this project is a list of 55 new SNPs to be used for ancestry identification in the future as well as a possible new classification method to use when performing ancestral identification. From these 55 SNPs two reduced panels were obtained; namely a 18-SNP panel produced by the impact analysis, and a 22-SNP panel made up of those SNPs available in the real data. Their respective performances are shown in the real and simulated data.

\subsubsection{Forensic Implications}

In regards to introducing this material to the wider forensic community the point is mute due to others already performing research into this area. The technology met with initially mixed results due to the implications of racial profiling in certain parts of the world. This research could be used by these people to increase the effectiveness of their genocidal plans. This fear is a possibility while the benefits of this research are more concrete. The profiling will allow for more focused searches by law enforcement when determining what individuals to question in regard to a DNA sample found at a crime scene.

This thesis does add to the general knowledge base by providing a set of SNP panels (55SNP, 22-SNP, and 18-SNP panels) that can be used for ancestral classification. Using the novel SNPs related to soft biometrics helps link this work with current biometric research. This panel of SNPs can be used to focus on a certain portion of the population as being involved in the crime. The profiling cannot however be used in conviction since the SNPs are meant to classify between ancestral groups. Looking at the available data shows that at these locations multiple individuals from the same ancestral group have a high probability of having the same SNPs, meaning the SNP panels as proposed cannot be used for identification of an individual. 


\subsection{Future Work}

There is the possibility of using a wider panel of SNPs but limiting the total number of SNPs used for a certain classifier. This would remove the SNPs performing poorly for certain ancestry groups causing incorrect classifications for these groups. While we think that this may lead to better classification results, the primary problem of classification errors may not, in fact, be reduced. Certain ancestry groups are so similar across many of the SNPs included in the profiles; this could limit the effectiveness in classification.

Another area in need of more work is the creation of a larger data set. Other groups overcame this problem by using their own genetic mappings. Gaining access to this data is one of the possible solutions to this problem. The only other solution to solving this problem is to create our own data set to use for testing. There is currently work being done by our group at West Virginia University to provide some of the needed data for testing new classification systems for ancestral origin.

One other possible idea for improving the classification system involves using multiple SNP panels separately. Each ancestral group would be given a unique SNP panel that works extremely well for that group. Then each individual would be compared to the four new panels and whichever panel provides the best results would be the group the individual is classified as. This is one line of work that could improve the classification rate, thought at a potentially more computational cost.

The final area for improvement we are considering is using the Kullback-Leibler distance metric to select possible SNPs to use. The KL distance makes use of distributions, which are what our SNP profiles are made of. The metric, however, does not work well with distributions that contain zeroes so we will have to substitute in an extremely small number. This will allow the metric to work regardless of the distribution used. 


\section{References}

1. Frudakis, T. et al. A Classifier for the SNP-Based Inference of Ancestry. J Forensic Sci. (2003) July; 48(4): 1-12

2. Wetton, $\mathbf{J}$ et al. Inferring the population of origin of DNA evidence within the UK by allele specific hybridization of Y-SNPs. Forensic Science International (2005) May; 152: 45-53

3. Kidd, J. et al. Analysis of a set of 128 ancestry informative single-nucleotide polymorphisms in a global set of 119 population samples. Investigative Genetics (2011)

4. Ross, A. and A.K. Jain, "Human Recognition Using Biometrics: An Overview," Annals of Telecommunications (2007) Jan/Feb; 62(1/2):11-35

5. Jain, A.K., A. Ross and S. Prabhakar, " An Introduction to Biometric Recognition", IEEE Transactions on Circuits and Systems for Video Technology, Special Issue on Image- and Video-Based Biometrics(2004) Jan; 14(1): 4-20

6. Adjeroh, D., D. Cao, M. Piccirilli, A. Ross, "Predictability and Correlation in Human Metrology", Proc. of IEEE International Workshop on Information Forensics and Security, (Seattle, USA), (2010) Dec.

7. Guo, G., Y. Fu, and T. S. Huang, “Age synthesis and estimation via faces: A survey,” IEEE Trans. Pattern Anal. Mach. Intell. (2010) Nov; 32(11):1955-1976

8. Cao, D., D. Adjeroh, M. Piccirilli, A. Ross, "Predictability and Correlation in Human Metrology," Proc. of IEEE International Workshop on Information Forensics and Security (WIFS), (Seattle, USA), (2010) Dec

9. Ross, A., R. Jillela, J. Smereka, V. N. Boddeti, B. V. K. VijayaKumar, R. Barnard, X. Hu, P. Pauca, R. Plemmons, "Matching Highly Non-ideal Ocular Images: An Information Fusion Approach," Proc. of the 5th IAPR International Conference on Biometrics (ICB), (New Delhi, India), (2012) Mar/Apr

10. Iannarelli, A. V. Ear identification. Fremont, Calif.: Paramont Pub. Co (1989) 
11. Graydon, M. et al. Inferring ethnicity using 15 autosomal STR loci-Comparisons among populations of similar and distinctly different physical traits. Forensic Science International: Genetics (2009); 3:251-54

12. Kayser, M et al. DNA-based prediction of human externally visible characteristics in forensics: Motivations, scientific challenges, and ethical considerations. Forensic Science International: Genetics (2009); 3:154-61

13. Watson J.D., F.H.C. Crick. "A Structure for Deoxyribose Nucleic Acid". Nature (1953); 171 (4356): 737-738.

14. International Human Genome Sequencing Consortium. "Finishing the euchromatic sequence of the human genome". Nature (2004); 431 (7011): 931-45.

15. Neel J.V., P.E. Smouse. Multivariate analysis of gametic disequilibrium in the Yanomama. Genetics (1977) Apr; 85(4): 733-52.

16. Chakraborty, R. Multiple alleles and estimation of genetic parameters: computational equations showing involvement of all alleles. Genetics (1992); 130: 231-43.

17. Shriver M.D., M.W. Smith, J. Lin, A. Marcini, J.M. Akey, R. Deka. Ethnic-affiliation estimation by use of population-specific DNA markers. Am J Hum Genet (1997) Apr; 60(4): 957-64

18. Nassir, R. et al. An ancestry informative marker set for determining continental validation and extension using human genome diversity panels. BMC Genet (2009); 10:39

19. Lowe, A., A. Urquhart, L.A. Foreman, I.W. Evett. Inferring ethnic origin by means of an STR profile. Forensic Sci Int (2001) Jun; 119(1):17-22

20. Syndercombe-Court, D., D. Ballard, C. Phillips, A. Revoir, C. Robson, C. Thacker. Comparison of Y chromosome haplotypes in three racial groups and the possibility of predicting ethnic origin. Int. Congress Ser. (2003); 1239:67-69

21. Egeland, T. et al. Inferring the Most Likely Geographical Origin of mtDNA Sequence Profiles. Annuls of Human Genetics (2004); 68: 461-471 
22. Ross, A., A. Abaza, "Human Ear Recognition," IEEE Computer, November 2011.

23. Brenner, C.H. Difficulties in the estimation of ethnic affiliation. Am J Hum Genet (1998) Jun; 62(6):1558-60.

24. Cavalli-Sforza, L. Luca et al. Opinion: The Human Genome Diversity Project: past, present and future. Nature Reviews Genetics (2005); 6(4): 333-40. 